\title{
A COMPARATIVE STUDY BETWEEN DIFFERENT SURGICAL TECHNIQUES FOR PREAURICULAR SINUS AT TERTIARY CARE CENTRE, SECUNDERABAD, TELANGANA
} \author{
Novshaba6, Abhinav Thaduri7, Dipin Kumar $V^{8}$ \\ ${ }^{1}$ Assistant Professor, Department of Otorhinolaryngology, Gandhi Medical College.
${ }^{2}$ Senior Resident, Department of Otorhinolaryngology, Gandhi Medical College.
3Postgraduate Student, Department of Otorhinolaryngology, Gandhi Medical College.
4Professor, Department of Otorhinolaryngology, Gandhi Medical College.
${ }^{5}$ Assistant Professor, Department of Otorhinolaryngology, Gandhi Medical College.
${ }^{6}$ Postgraduate Student, Department of Otorhinolaryngology, Gandhi Medical College.
7Postgraduate Student, Department of Otorhinolaryngology, Gandhi Medical College.
8Postgraduate Student, Department of Otorhinolaryngology, Gandhi Medical College.

${ }^{8}$ Postgraduate Student, Department of Otorhinolaryngology, Gandhi Medical College.

Ahmed Abdul Khabeer1, Ivaturi Phani Bhushan², Rathod Sunil Kumar ${ }^{3}$, A. V. S. Hanumantha Rao 4 , Kamanur Samson Deva Kumar ${ }^{5}$,

\section{BACKGROUND}

ABSTRACT

Preauricular sinus is an asymptomatic congenital abnormality of external ear found along the ascending crux of the helix or adjacent to tragus. Asymptomatic preauricular sinus does not require any intervention. The most common clinical presentation of infected preauricular sinus is erythema, swelling, pain and discharge. Infected preauricular sinus requires surgical excision to prevent recurrence and reinfection.

The objective of this study is to compare the outcomes of preauricular sinus excision by classic simple sinusectomy, classic simple sinusectomy with methylene blue dye injection and supra-auricular approach.

\section{MATERIALS AND METHODS}

A prospective study was carried out in Department of Otorhinolaryngology of Gandhi Medical College, Secunderabad from 2009 to 2014 with a 3-year follow-up period. Patients were evaluated on the outcomes of 3 surgical techniques namely classic simple, sinusectomy, classic simple sinusectomy with methylene blue injection and supra-auricular approach.

\section{RESULTS}

A total of 50 patients required surgical excision of preauricular sinus during study period. Around $64 \%$ of cases were in the age group of $6-10$ years. Around 15\% of study population who underwent classic simple sinusectomy showed recurrence after excision and $10 \%$ of subjects who underwent classic simple sinusectomy with intraoperative methylene blue injection showed recurrence. There were no recurrences reported among study subjects who underwent excision of sinus by supra-auricular approach.

\section{CONCLUSION}

From the present study, it can be concluded that supra-auricular approach has no recurrence/ least recurrence when compared with other surgical techniques for management of preauricular sinus.

\section{KEYWORDS}

Preauricular Sinus, Classic Simple Sinusectomy, Classic Simple Sinusectomy with Methylene Blue Injection, Supra-auricular Approach.

HOW TO CITE THIS ARTICLE: Khabeer AA, Bhushan IP, Kumar RS, et al. A comparative study between different surgical techniques for preauricular sinus at tertiary care centre, Secunderabad, Telangana. J. Evolution Med. Dent. Sci. 2018;7(04):474478, DOI: $10.14260 /$ jemds/2018/106

\section{BACKGROUND}

Preauricular sinus/ pit is a common congenital malformation seen in $0.3 \%-0.9 \%$ of the population. It is present as a pit or depression at the anterior margin of ascending limb of helix, adjacent to the root of helix or adjacent to the tragus. Preauricular sinus affects both sexes equally. It is inherited as an autosomal dominant incomplete pattern with variable power of expression. ${ }^{1}$ Preauricular sinuses or cysts are found lateral and superior to the facial nerve and parotid gland.

'Financial or Other Competing Interest': None.

Submission 14-12-2017, Peer Review 07-01-2018,

Acceptance 13-01-2018, Published 22-01-2018.

Corresponding Author:

Dr. Ivaturi Phani Bhushan,

Plot No.131, H. No. 4-9-433, RTC Colony,

Hayathnagar, Hyderabad-501505.

E-mail: drphani21@gmail.com

DOI: $10.14260 /$ jemds $/ 2018 / 106$

\section{(c) $(1) \$$}

Around $50 \%$ of preauricular sinus cases involve the right side. ${ }^{2}$ Preauricular sinus/ pits are usually asymptomatic and when symptomatic present as a discharging sinus associated with abscess formation or associated with multiple scars due to repeated drainage usually seen anterior to the tragus. ${ }^{1}$ Recurrent or persistent preauricular sinus infection requires surgical excision of the sinus along with its tract during a period of quiescence. ${ }^{2}$

Various surgical techniques have been described with variable results. Incomplete excision results in increased recurrence. The standard technique is classic simple sinusectomy, that is to excise an ellipse of skin surrounding the preauricular sinus opening and to dissect out the individual tract. ${ }^{2}$ Second technique is classic simple sinusectomy using methylene blue. In this procedure methylene blue is injected into the tract intraoperatively, tract is identified, dissection is done around the tract and is excised. The supra-auricular technique involves wide local 
excision by extended post-auricular incision under general anaesthesia. The supra-auricular approach has been giving good results. ${ }^{2}$ Soft tissue between the plane of the temporalis fascia and the skin anterior to the sinus is excised along with a piece of adjoining helical cartilage. This excised soft tissue contains the entire tract with its branches giving no room for recurrence, ensuring complete removal of the tract. ${ }^{1}$

\section{Objective}

To compare the outcomes of preauricular sinus excision by classic simple sinusectomy, classic simple sinusectomy with methylene blue dye injection and supra-auricular approach.

\section{MATERIALS AND METHODS \\ Study Design}

Observational study to evaluate outcomes of classic simple sinusectomy with methylene blue and supra-auricular approach for excision of preauricular sinus and compare it with classic simple sinusectomy.

\section{Study Area}

Department of Otorhinolaryngology, Gandhi HospitalTertiary Care Centre, Secunderabad, Telangana.

\section{Study Duration}

5 years (2009 - 2014). All the cases were followed up for a period of 3 years. Hence, the study concluded in 2017 including the follow-up period of last case.

\section{Sample Size}

This study proposed to collect data prospectively of all patients admitted with preauricular sinus requiring surgical excision from 2009 - 2014. A total of 50 patients with preauricular sinus were admitted in the hospital during the study period. All the 50 patients were included in the study. Among these 50 patients 20 sinuses were excised with classic simple sinusectomy technique, another 20 sinuses were excised by supra-auricular approach, i.e. wide local excision by extended post-auricular incision and 10 sinuses were excised using classic simple sinusectomy with methylene blue approach among which preauricular sinus was excised by injecting methylene blue dye intraoperatively, identifying the tract and by dissecting around the tract and excising the dissected area. Outcomes for all the 3 techniques in terms of recurrence rate were observed. A thorough history was taken, and complete head and neck examination was performed for all patients. Surgeries were conducted in an infection-free interval and under local anaesthesia. All the cases were followed up post-operatively at week 1 , once every 2 weeks for 1 month, monthly once for 6 months and then once every 3 months for 3 years.

\section{Exclusion Criteria}

Asymptomatic preauricular sinus patients and patients who were not willing to take part in the study were excluded.

\section{Data Analysis}

Data was analysed using MS Excel and SPSS v 17.0. Results are interpreted in terms of percentages and Fisher exact test of significance was applied wherever required.

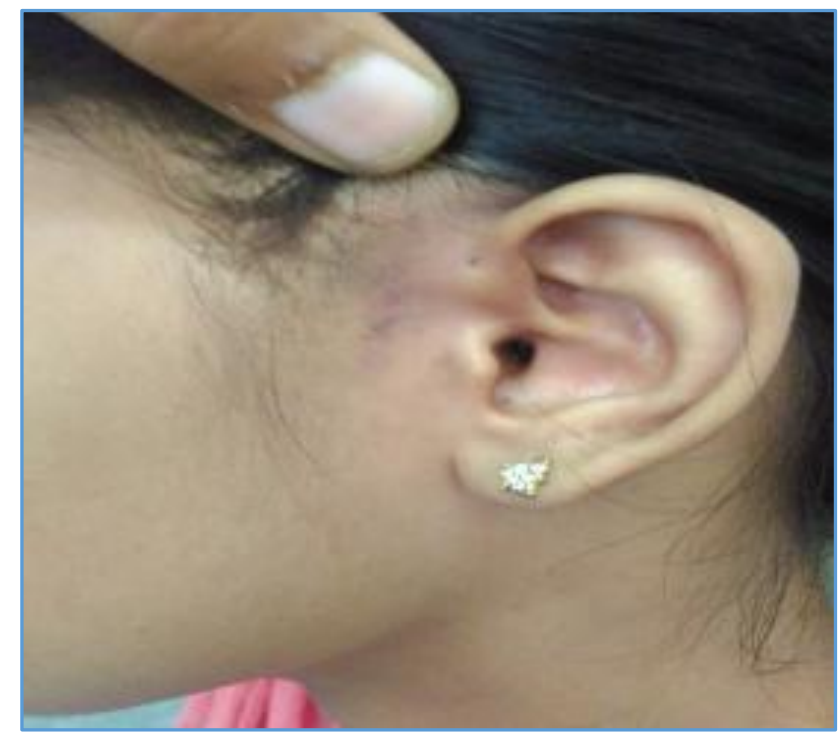

Figure 1. Pre Auricular Sinus

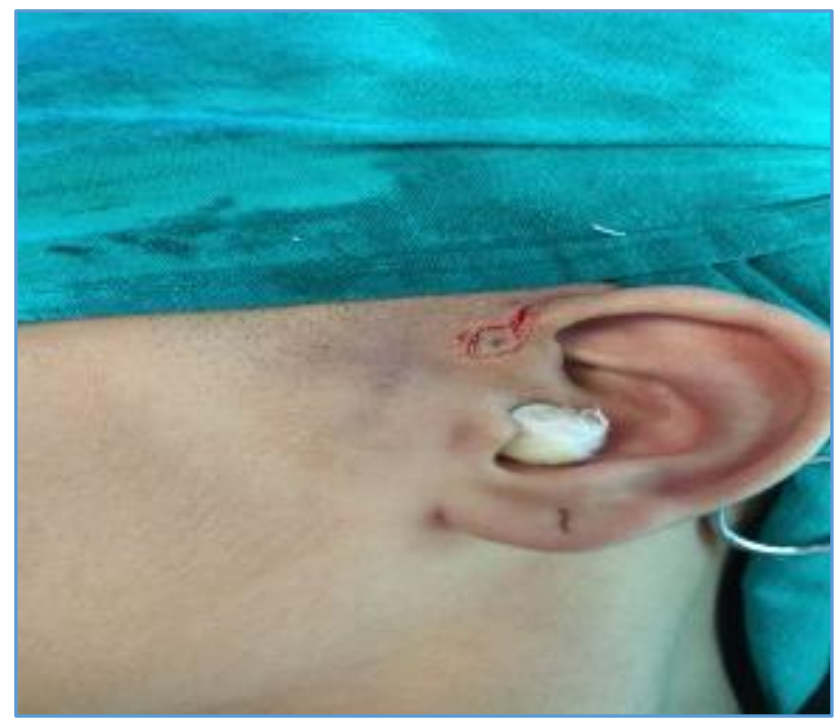

Figure 2. Incision with Supra Auricular Extension

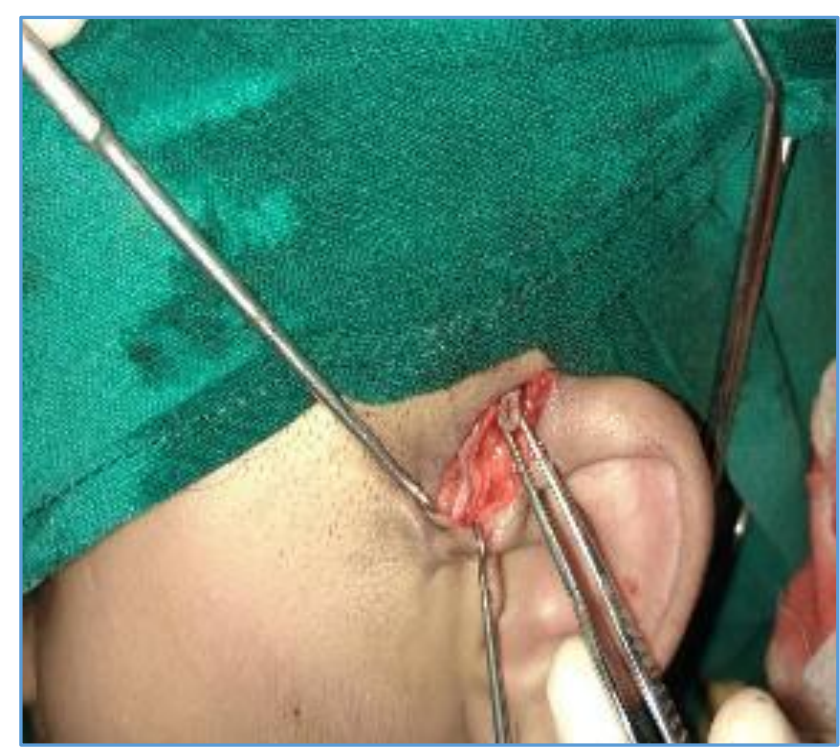

Figure 3. Dissecting the Tract 


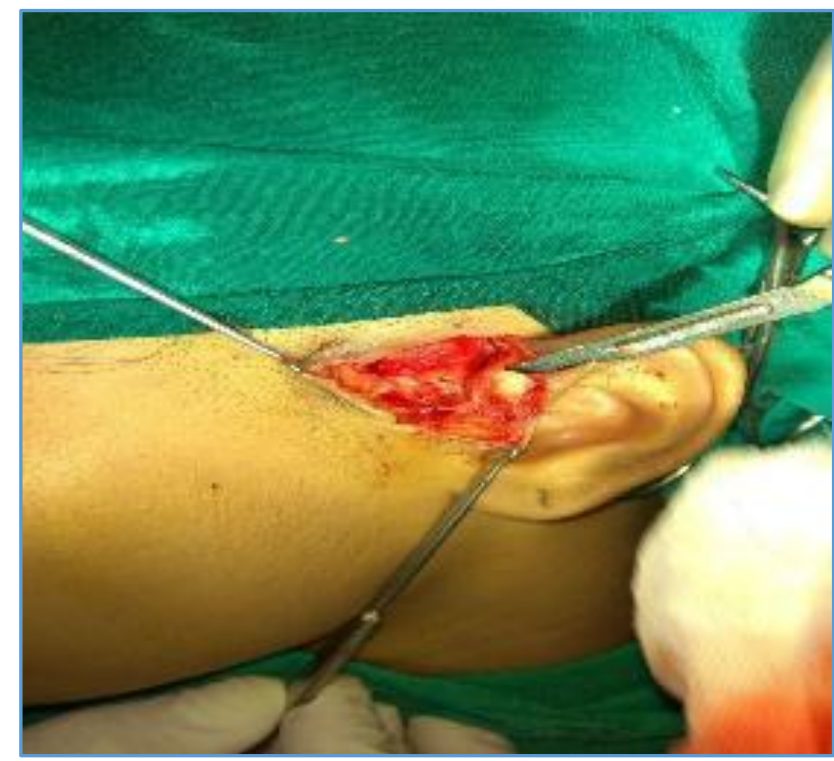

Figure 4. Removal of Soft Tissue

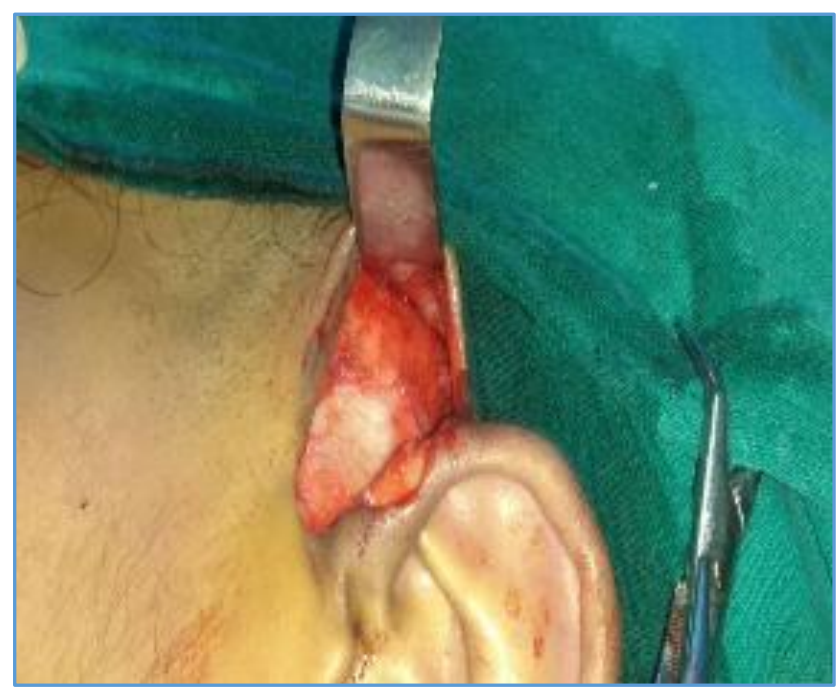

Figure 5. Complete Excision of Tissue between skin and Temporalis Fascia

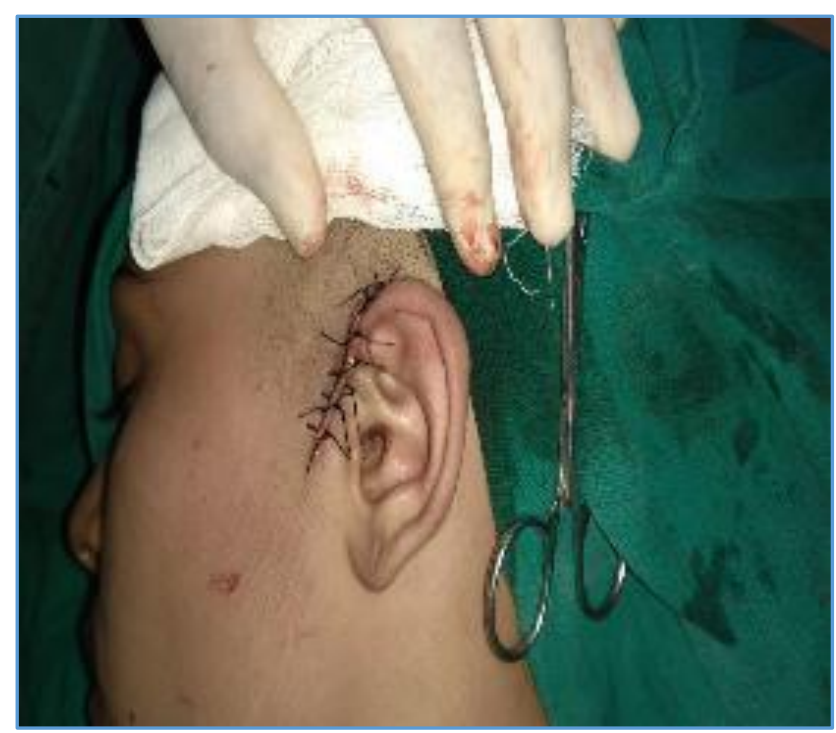

Figure 6. Closure with 3'0 silk

\section{RESULTS}

Fifty patients were operated for symptomatic preauricular sinus. Around $64 \%$ of study participants belonged to age group of 6 - 10 years, $28 \%$ were in the age group of $11-15$ years and $8 \%$ were in the age group of $16-20$ years.

\begin{tabular}{|c|c|c|}
\hline Age Group & Number & $\mathbf{\%}$ \\
\hline $6-10$ & 32 & 64 \\
\hline $11-15$ & 14 & 28 \\
\hline $16-20$ & 4 & 8 \\
\hline Total & $\mathbf{5 0}$ & $\mathbf{1 0 0}$ \\
\hline Gender & Number & $\mathbf{\%}$ \\
\hline Male & 12 & 24 \\
\hline Female & 38 & 76 \\
\hline Total & $\mathbf{5 0}$ & $\mathbf{1 0 0}$ \\
\hline Laterality & Number & $\mathbf{\%}$ \\
\hline Right & 40 & 80 \\
\hline Left & 6 & 12 \\
\hline Bilateral & 4 & 8 \\
\hline Total & $\mathbf{5 0}$ & $\mathbf{1 0 0}$ \\
\hline
\end{tabular}

Table 1. Distribution of Study Population according to Age, Gender and Site of Preauricular Sinus

In the present study, it was found that $76 \%$ of study subjects were females and $24 \%$ of males had preauricular sinus. Around $80 \%$ of study subjects had a preauricular sinus on right side and $12 \%$ on left side. Eight percent of them had bilateral sinuses. In the present study there were 4 cases with bilateral sinuses, but the total number of sinuses operated were 50 only. Among cases who had bilateral sinuses, unilateral excision was done as the other sinus was asymptomatic.

\begin{tabular}{|c|c|c|}
\hline Surgical Technique & $\begin{array}{c}\text { Number } \\
(\%)\end{array}$ & \begin{tabular}{|c|} 
Recurrence \\
$(\%)$
\end{tabular} \\
\hline Classic Simple Sinusectomy & $20(40)$ & $3(6)$ \\
\hline $\begin{array}{l}\text { Classic Simple Sinusectomy with } \\
\text { Methylene Blue }\end{array}$ & $10(20)$ & $1(2)$ \\
\hline Supra-auricular approach & $20(40)$ & $0(0)$ \\
\hline Total & $50(100)$ & $4(8)$ \\
\hline
\end{tabular}

Twenty patients underwent excision by simple sinusectomy and another 20 patients were operated by supra-auricular approach. Ten patients were excised of their sinus tract by simple sinusectomy with methylene blue.

Total recurrence rate in the present study was found to be $8 \%$. Around $6 \%$ of recurrence was found with classic simple sinusectomy procedure and $2 \%$ recurrence was found with Classic simple sinusectomy with methylene blue procedure. There were absolutely no recurrences among those who underwent excision by supra-auricular approach.

\begin{tabular}{|c|c|c|c|}
\hline : & 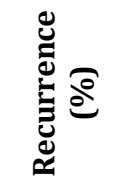 & 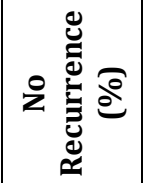 & 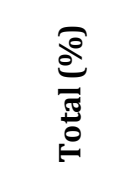 \\
\hline Classic Simple Sinusectomy & $3(15)$ & $17(85)$ & $20(100)$ \\
\hline $\begin{array}{l}\text { Classic Simple Sinusectomy } \\
\text { with Methylene Blue }\end{array}$ & $1(10)$ & $9(90)$ & $10(100)$ \\
\hline Total & $4(13.3)$ & $26(86.7)$ & $30(100)$ \\
\hline
\end{tabular}

Table 3. Recurrence among Patients who Underwent

Simple Sinusectomy and Simple Sinusectomy with Methylene Blue 
Among the patients who underwent simple sinusectomy (20 cases) 3 cases showed recurrence (15\%) and among those who underwent sinusectomy with methylene blue (10 cases) 1 case showed recurrence (10\%). Using Fisher exact test of significance, $p$ value was found as $>0.05$ (statistically not significant).

\begin{tabular}{|c|c|c|c|}
\hline 节 & 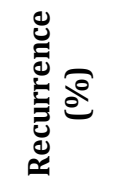 & 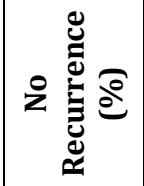 & 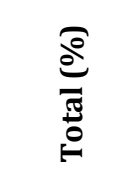 \\
\hline Classic Simple Sinusectomy & $3(15)$ & $17(85)$ & $20(100)$ \\
\hline Supra-auricular Approach & $0(0)$ & $20(100)$ & $20(100)$ \\
\hline Total & $3(7.5)$ & $37(92.5)$ & $40(100)$ \\
\hline \multicolumn{4}{|c|}{$\begin{array}{l}\text { Table 4. Recurrence among Patients who Underwent } \\
\text { Simple Sinusectomy and Excision by Supra-auricular } \\
\text { Approach }\end{array}$} \\
\hline
\end{tabular}

Among the patients who underwent simple sinusectomy (20 cases) 3 cases showed recurrence (15\%) and among those who underwent excision by supra-auricular approach (20 cases) no recurrences were reported. Using Fisher exact test of significance, $\mathrm{p}$ value was found to be $<0.05$ (statistically significant).

\begin{tabular}{|c|c|c|c|}
\hline 莺 & 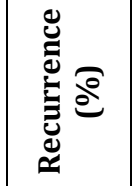 & 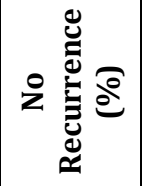 & 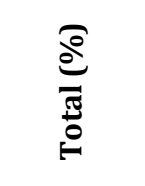 \\
\hline $\begin{array}{c}\text { Classic Simple Sinusectomy + } \\
\text { Classic Simple Sinusectomy } \\
\text { with Methylene Blue } \\
\end{array}$ & $4(13.3)$ & $26(86.7)$ & $30(100)$ \\
\hline Supra-auricular Approach & $0(0)$ & $20(100)$ & $20(100)$ \\
\hline Total & $4(8)$ & $46(92)$ & $50(100)$ \\
\hline \multicolumn{4}{|c|}{$\begin{array}{c}\text { Table 5. Recurrence among Patients Who Underwent } \\
\text { Simple Sinusectomy, Sinusectomy with Methylene Blue and } \\
\text { Excision by Supra-Auricular Approach }\end{array}$} \\
\hline
\end{tabular}

In the present study, patients who were excised by supraauricular approach and those who underwent other surgical techniques were compared for recurrence and it was found that recurrence rate in classic simple sinusectomy and sinusectomy with methylene blue was found to be statistically significant. (Using Fisher exact test of significance, $p$ value was found to be $<0.05$ ). The present study findings were similar to the case reports reported by Mathew Clarke et al,3 where wide excision was associated with least recurrence rate.

\section{DISCUSSION}

The study findings with relation to age distribution were similar to a study conducted by Vijayendra et $\mathrm{al}^{1}$ and $\mathrm{M}$ Attallah ${ }^{4}$ where maximum incidence of preauricular sinus was in the age group of 1 - 10 years. The study findings differed with a study conducted by Richa Gupta et al, 5 where $42.3 \%$ of cases were found in the age group of $11-20$ years. The present study findings in relation to gender and laterality of sinus were similar to a study conducted by ElAassar et al, 6 where $67 \%$ of study subjects were females and $46.5 \%$ of them had right preauricular sinus. Richa Gupta et al ${ }^{5}$ findings were different from present study, where left preauricular sinus (46.15\%) was slightly commoner than right preauricular sinus $(42.3 \%)$.

Overall, recurrence rate in the present study was found to be $8 \%$. Around $6 \%$ of recurrence was found with classic simple sinusectomy procedure and $2 \%$ recurrence was found with classic simple sinusectomy with methylene blue procedure. Zero recurrence was found among those who underwent excision by supra-auricular approach. Similar findings were found in a study by $\mathrm{H}$ Vijayendra et al, ${ }^{1}$ where there were no recurrences with supra-auricular approach.

In the present study it was found that among the patients who underwent simple sinusectomy (20 cases) recurrence rate was $15 \%$ and among those who underwent sinusectomy with methylene blue (10 cases) recurrence rate was $10 \%$. The present study findings were concurrent with a study conducted by ElAasaar et al, 6 where recurrence was highest with simple sinusectomy (28\%).

In this study, among the patients who underwent simple sinusectomy (20 cases) recurrence rate was $15 \%$ and among those who underwent excision by supra-auricular approach (20 cases) no recurrences were reported. The difference was found statistically significant $(\mathrm{p}<0.05)$. The present study findings concurred with ElAassar et al $^{6}$ where only one case showed recurrence with supra-auricular approach and the difference was found significant statistically. Similar findings were found in a study by Lam et al,7 where supra-auricular approach was found to have least recurrence compared to simple sinusectomy.

In the present study, it was found that supra-auricular approach had no recurrences when compared to any other surgical approach and the difference was found to be statistically significant $(\mathrm{p}<0.05)$. The present study findings were similar to the case reports reported by Mathew Clarke et $\mathrm{al}^{3}{ }^{3}$ where wide excision was associated with least recurrence rate.

\section{CONCLUSION}

From the present study it can be concluded that supraauricular approach has the best clinical outcome when compared to classic simple sinusectomy and simple sinusectomy with methylene blue for the treatment of preauricular sinus. Cause of recurrence in classic simple sinusectomy and classic simple sinusectomy with methylene blue could be due to incomplete excision of tract/ branches of the tract. Though probing and dye injection was done to delineate the tract clearly, but still there were recurrences which indicated that finer branches might get missed and cause recurrence.

Supra-auricular approach involves excision of soft tissue containing the entire tract with its branches, giving no room for recurrence, ensuring complete removal of the tract.

Supra-auricular approach had significantly no recurrence when compared with other tract sinusectomy approaches, so from current study it can be recommended to recognise supra-auricular approach of excision as standard primary procedure for preauricular sinus excision.

\section{ACKNOWLEDGEMENTS}

The authors are grateful to all the patients who have been part of the study and administration of Gandhi Hospital for allowing to carry out the research study. 


\section{REFERENCES}

[1] Vijayendra H, Sangeetha R, Chetty KR. A safe and reliable technique in the management of preauricular sinus. Indian J Otolaryngol Head Neck Surg 2005;57(4):294-5.

[2] Chowdary KVSK, Chandra NS, Madesh RK. Preauricular sinus: a novel approach. Indian J Otolaryngol Head Neck Surg 2013;65(3):234-6.

[3] Clarke M, Amin K, Rahul K, et al. A pinna helix sinus: a developmental anomaly. The Otorhinolaryngologist 2013;6(3):180-1.

[4] Attallah M. Management of congenital preauricular sinuses 5 years' experience. Indian Journal of Otolaryngology and Head and Neck Surgery 1993;45(3):143-4.
[5] Gupta R, Agrawal A, Poorey VK. Pre auricular sinus: a clinicopathological study. Int J Res Med Sci 2015;3(11):3274-7.

[6] ElAassar AS, Abd-El Hady M, Askar SM, et al. Preauricular sinus: a comparative study between different surgical approaches. Indian J Otol 2017;23(3):193-6.

[7] Lam HC, Soo G, Wormald PJ, et al. Excision of the preauricular sinus: a comparison of two surgical techniques. Laryngoscope 2001;111(2):317-9. 